\title{
ACQUISITION OF GEOMETRICAL DATA OF SMALL RIVERS WITH AN UNMANNED WATER VEHICLE
}

\author{
Hannes Sardemann*, Anette Eltner, Hans-Gerd Maas
}

Institute of Photogrammetry and Remote Sensing, Technische Universität Dresden, Germany
(hannes.sardemann, anette.eltner, hans-gerd.maas)@tu-dresden.de

\section{Commission II, ICWG I/II}

KEY WORDS: Unmanned Water Vehicle, Multi-sensor Platform, Mobile Mapping, Lidar, Echo Sounder, Flash Flood

\begin{abstract}
:
Rivers with small- and medium-scaled catchments have been increasingly affected by extreme events, i.e. flash floods, in the last years. New methods to describe and predict these events are developed in the interdisciplinary research project EXTRUSO. Flash flood events happen on small temporal and spatial scales, stressing the necessity of high-resolution input data for hydrological and hydrodynamic modelling. Among others, the benefit of high-resolution digital terrain models (DTMs) will be evaluated in the project.

This article introduces a boat-based approach for the acquisition of geometrical and morphological data of small rivers and their banks. An unmanned water vehicle (UWV) is used as a multi-sensor platform to collect 3D-point clouds of the riverbanks, as well as bathymetric measurements of water depth and river morphology. The UWV is equipped with a mobile Lidar, a panorama camera, an echo sounder and a positioning unit. Whole (sub-) catchments of small rivers can be digitalized and provided for hydrological modelling when UWV-based and UAV (unmanned aerial vehicle) based point clouds are fused.
\end{abstract}

\section{INTRODUCTION}

In the last decades, an increasing number of high-intensity rainfall events has been observed in central Europe (Mueller and Pfister, 2011). This results in a higher risk for flash-flood events, especially in rivers with small catchments. Flash floods develop in a short time and on a small scale, making them difficult to monitor and predict with traditional methods (Borga et al., 2008). Therefore, new hydrological and hydrodynamic models have to be developed and existing models have to be adapted in order to be able to predict flash floods. These models require input data with high temporal and spatial resolution. In this article, a system for the acquisition of high-resolution point clouds is presented.

A remote controlled UWV, equipped with multiple sensors for mapping its surroundings above and underneath the water level, is presented. In combination with UAV-based point clouds, which cover a broader area of the river catchment, DTMs of subcatchments can be generated. This can serve as an input for hydrodynamic modelling and for the prediction of flooded areas. Furthermore the data can be used for post event analysis.

\section{THE UNMANNED WATER VEHICLE}

The boat platform used in this study is the remote controlled survey boat HyDrone by Seafloor Systems. The catamaran was originally designed for bathymetric surveys and has been rebuilt by the authors to the needs of a multisensor-platform (Figure 1). The platform has a maximum speed of $20 \mathrm{kts}$, a payload of $11.3 \mathrm{~kg}$ and a battery endurance of 5-8 hours, depending on the survey speed. The batteries are placed inside the pontoons, supplying power for the thrusters as well as for the attached sensors.

\subsection{Components}

Above water level, the mobile lidar Velodyne Puck scans the riverbanks in 16 profiles with up to 0.3 million points per second and a maximum range of $100 \mathrm{~m}$. The 3D point accuracy is $+/-3 \mathrm{~cm}$. A Samsung Gear 360 records a panoramic video of the survey that can be used for point cloud colorization. The $360^{\circ}$ camera consist of two fisheye lenses with a field of view of $>180^{\circ}$ each, pointing to opposite directions, enabling a full panorama with 7.3 megapixels. The positioning unit is the GNSS-supported IMU Spatial by Advanced Navigation. It has $0.6 \mathrm{~m}$ horizontal, $1 \mathrm{~m}$ vertical, and $0.2^{\circ}$ rotation angle accuracy. The single-beam echo sounder EchoLogger ECT400 measures water depth with $10 \mathrm{~Hz}$ maximum ping rate. For sensor control and data storage, two RaspberryPi computers are deployed on the boat. The collected data is processed after the survey in the office.

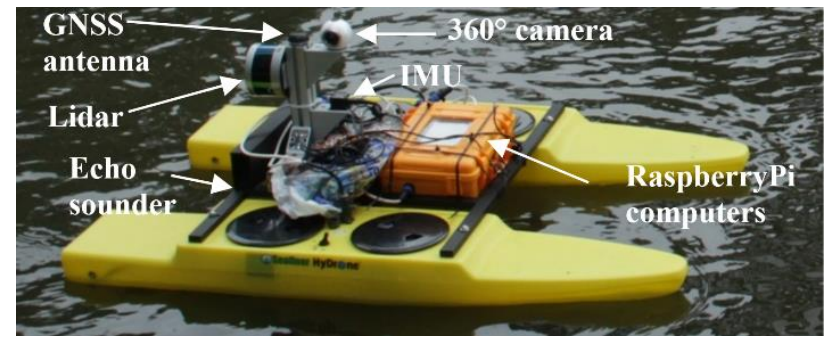

Figure 1. Multi Sensor UWV with Lidar, $360^{\circ}$ camera, echo sounder (hidden) and GNSS-supported IMU

\subsection{Time Synchronisation}

In order to enable data fusion between the sensors, they need to have synchronized clocks. The IMU clock is synchronized with GPS time with $50 \mathrm{~ns}$ accuracy and serves as time reference. It sends a time stamp every second to the Velodyne lidar via RS232 interface. The lidar's internal clock is $0.05 \mathrm{~ns}$ accurate and assigns a time stamp to every $3 \mathrm{D}$ point measurement. The echo sounder transmits its data also via RS232 to the IMU, where it is stored and a time stamp is assigned. Time synchronisation is more challenging for the consumer camera Samsung Gear 360, since it is not possible to trigger the camera externally. One

\footnotetext{
* Corresponding author
} 
approach is to attach a display to the boat, showing the IMU time with a high refresh rate. Having the full spherical view of the $360^{\circ}$ camera, a spot can be found, where the display is always in the camera's field of view and does not conclude the areas of interest, i.e. the riverbanks. The time stamp can be read manually or with optical character recognition for every frame. First tests revealed that this approach works well in indoor environments, but fails when the sun reflects from the display.

\subsection{Calibration}

Besides time synchronization, calibration of the relative orientations is crucial for data fusion and point cloud colorization. The calibration procedure introduced by Mader et al. (2014) was adapted for that purpose. The used calibration field consists of seven cones with coded markers on their surface. A reference point cloud of the calibration field was created with bundle adjustment using a SLR camera, scale bars and the coded markers. A dense point cloud was created, using structure from motion (SfM) and the results of the marker-based bundle adjustment (Figure 2a).

The UWV is placed in the middle of the calibration field, so that all cones are inside the fields of view of both lidar and $360^{\circ}$ camera (Figure 2b-c). The exterior orientation of both sides of the camera are determined using marker-based space resection, applying a fisheye camera model. Interior orientation parameters were calibrated prior and then fixed. The $3 \mathrm{D}$ reference points are transformed from object space to the camera coordinate system:

$$
\mathbf{X}_{\text {cam }}=\mathbf{R}_{\text {ref }}^{\text {cam }} \cdot\left(\mathbf{X}_{\text {ref }}-\mathbf{X}_{\text {ref }}^{\mathbf{0}}\right)
$$

where: $\quad \mathbf{X}_{\mathrm{s}}=\left[\begin{array}{l}x_{s} \\ y_{s} \\ z_{s}\end{array}\right]=$ point in specific coordinate system $\mathrm{s}$

$\mathbf{X}_{\text {ref }}^{\mathbf{0}}=$ coordinates of projection centre

$\mathbf{R}_{\mathrm{s} 1}^{s 2}=$ rotation matrix from coordinate system $\mathrm{s} 1$ to $\mathrm{s} 2$

The origin of the camera coordinate system lies in the projection centre with its axes parallel to image space. According to an equidistant fisheye model, camera coordinates are projected into image space:

$$
\begin{aligned}
& \mathrm{x}^{\prime}=\mathrm{x}_{\mathrm{o}}^{\prime}+\mathrm{c} \cdot \alpha \cdot \frac{\mathrm{x}_{\mathrm{cam}}}{\sqrt{\mathrm{X}_{\mathrm{cam}}^{2}+\mathrm{Y}_{\mathrm{cam}}^{2}}}+\Delta \mathrm{x}^{\prime} \\
& \mathrm{y}^{\prime}=\mathrm{y}_{0}^{\prime}+\mathrm{c} \cdot \alpha \cdot \frac{\mathrm{Y}_{\mathrm{cam}}}{\sqrt{\mathrm{X}_{\mathrm{cam}}^{2}+\mathrm{Y}_{\mathrm{cam}}^{2}}}+\Delta \mathrm{y}^{\prime}
\end{aligned}
$$

where: $x^{\prime}, y^{\prime}=$ image coordinates

$$
\begin{aligned}
& \mathrm{x}_{0}{ }^{\prime}, \mathrm{y}_{0}{ }^{\prime}=\text { principle point } \\
& \mathrm{c}=\text { focal length } \\
& \alpha=\text { incidence angle of image ray }
\end{aligned}
$$

In order to determine the relative orientation between camera and lidar, the exterior orientation of the lidar relative to the reference coordinate system has to be found:

$$
\mathbf{X}_{\text {ref }}=\mathbf{T}_{\text {lidar }}^{\mathrm{ref}}+\mathbf{R}_{\text {lidar }}^{\mathrm{ref}} \cdot \mathbf{X}_{\text {lidar }}
$$

where: $\quad \mathbf{T}_{\mathrm{s} 1}^{\mathrm{s} 2}=$ translation vector from coord. system s1 to s2
The transformation can be determined fitting cones in the reference and in the lidar point cloud (Figure 2d). Each cone is parameterized by its apex, the direction of its axis, and its opening angle. In a 'cone coordinate system', where the $\mathrm{z}_{\mathrm{c}}$-axis points along the cone axis and $\mathrm{x}_{\mathrm{c}}$ and $\mathrm{y}_{\mathrm{c}}$ are perpendicular to it, all points on the cone's surface follow the equation

$$
\left(x_{i, j}\right)_{c}^{2}+\left(y_{i, j}\right)_{c}^{2}-\left(z_{i, j}\right)_{c}^{2} \cdot \tan ^{2} \beta_{j}
$$

where: $\mathrm{x}_{\mathrm{c}}, \mathrm{y}_{\mathrm{c}}, \mathrm{z}_{\mathrm{c}}=$ surface points in cone coordinates

$$
\begin{aligned}
& \beta=\text { cone opening angle } \\
& \mathrm{i}=\text { point index }
\end{aligned}
$$

$\mathrm{j}=$ cone index

In order to fit cones in the reference point cloud, the surface points have to be transformed from reference coordinates to cone coordinates:

$$
\left(\mathbf{X}_{\mathrm{i}, \mathrm{j}}\right)_{\mathrm{c}}=\left(\mathbf{X}_{\mathrm{ap}, \mathrm{j}}\right)_{\mathrm{ref}}+\left(\mathbf{R}_{\mathrm{ref}}^{\mathrm{c}}\right)_{\mathrm{j}} \cdot\left(\mathbf{X}_{\mathrm{i}, \mathrm{j}}\right)_{\mathrm{ref}}
$$

where: $\quad \mathbf{X}_{\mathrm{ap}}=$ apex of cone

Since cones are rotation invariant around their main axis, only two rotation angles are needed to parameterize $\mathbf{R}_{\text {ref }}^{\mathbf{c}}$. Those angles determine the direction of the cone axis in the reference coordinate system. Together with the apex position, they build the five parameters of each cone. From equations (4), (5) and (6), the $(5 \cdot j)$ cone parameters and the relative orientation between lidar and reference coordinate system can be determined in a least squares adjustment.

Calibration of the relative orientation between IMU and sensors is a crucial requirement for the generation of a point cloud from a moving UWV. The translation vector, i.e. lever arm, is determined directly within the photogrammetric bundle adjustment by attaching a marker to the GNSS antenna. The boresight alignment can be simplified, because of the sensor configuration: IMU and lidar are attached to the same aluminium profile with the IMU pointing towards the front of the UWV and the lidar coordinate system being aligned perpendicular to it. The echo sounder is attached to the same profile. Since the accuracy of the echo soundings is expected to be in the range of a few centimetres, the offset was measured directly with a ruler. The calibration process will be extended with a lever arm and boresight-alignment calibration and a consideration of the echo sounder offset in future experiments. This will lead to an improved accuracy, especially when longer distances will be measured.

\section{DATA ACQUISITION}

First test surveys were conducted in a small river in Saxony, Germany. Segments of the river 'Freiberger Mulde' have been monitored in other research projects recently and an UAV based point cloud of the river surrounding areas already exists. Parts of the riverbanks are covered by trees and can therefore not be seen from the air. These gaps can be filled with UWV surveys. There is also no high-resolution model of the riverbed available yet, which can be recorded from UWV surveys. 


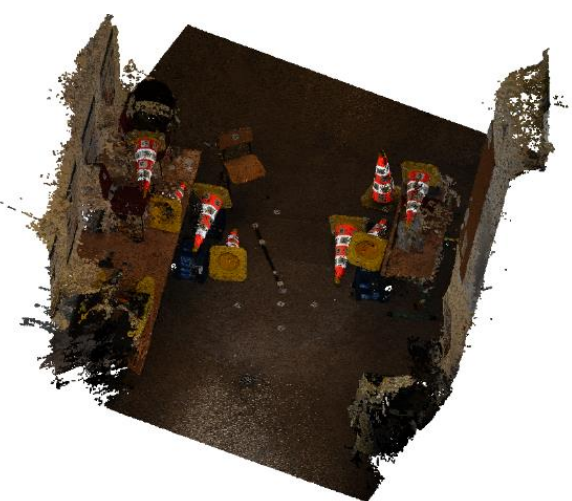

(a)

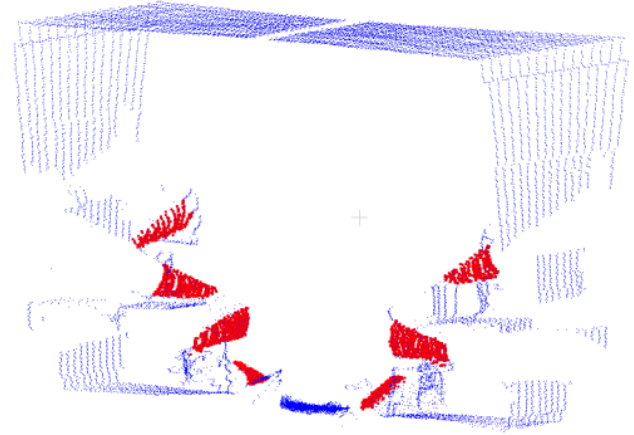

(c)

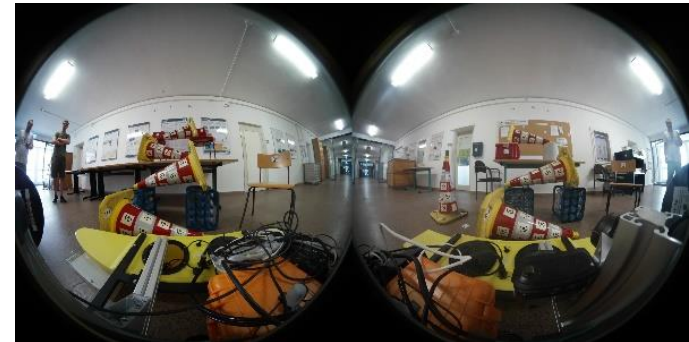

(b)

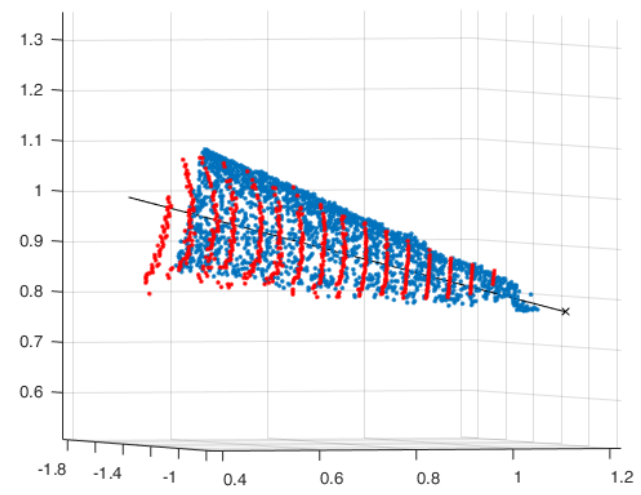

(d)

Figure 2. Calibration of relative orientations. The cone-based calibration field is measured with a SLR camera and used as reference (a). Markers are detected in the $360^{\circ}$ camera images and used for space resection (b). Cones are detected in the Lidar point cloud (c) and fitted with cones from the reference point cloud (d).

Considering the survey configuration, a good trade-off has to be found between a dense point coverage for the single beam echo sounder and a feasible point density of the lidar point cloud. Krüger et al. (2018) show that a good data acquisition strategy has to be chosen for the interpolation of single point echo soundings. A zigzag trajectory was implemented at the Freiberger Mulde (Figure 3).

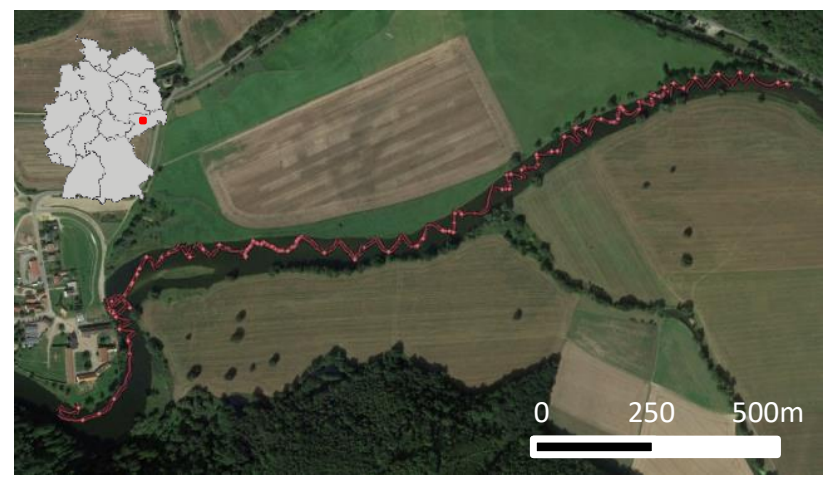

Figure 3. Survey configuration on the river Freiberger Mulde. The surveyed river segment is approx. $1.5 \mathrm{~km}$ long.

\section{DATA PROCESSING AND RESULTS}

During the UWV surveys, RaspberryPi computers are used to start the sensor recording and to store the data on a hard drive. The data is then post processed in the office.

\subsection{Lidar}

To gather a complete point cloud of the riverbanks, the lidar scanlines that were recorded along the river have to be combined according to the IMU position. In total, approx. 75 million points have been recorded. Boresight-Alignment and lever arm have to be considered in order to transform the recorded lidar points into the IMU coordinate system:

$$
\mathbf{X}_{\mathrm{IMU}}=\mathbf{T}_{\text {lidar }}^{\mathrm{IMU}}+\mathbf{R}_{\text {lidar }}^{\mathrm{IMU}} \cdot \mathbf{X}_{\text {lidar }}
$$

Since every point has a time stamp, they can be transformed into world coordinates, i.e. UTM:

$$
\mathbf{X}_{\mathrm{world}}=\mathbf{T}_{\mathrm{IMU}}^{\mathrm{world}}+\mathbf{R}_{\mathrm{IMU}}^{\mathrm{world}} \cdot \mathbf{X}_{\mathrm{IMU}}
$$

The $360^{\circ}$ camera images can be used for colorization of the point cloud. By projecting lidar points into image space of a frame recorded at a specific time, with known exterior and interior 
orientation, the colour value can be taken from the image. This projection follows equations (1) - (3).

Figure 4 shows the acquired lidar point cloud of the riverbanks. All points are shown either monochrome or intensity coloured because the $360^{\circ}$ camera has not been synchronized, yet. Figure 5 shows a more detailed view of a small segment on the northern bank including intensity values. The riverbank in the front is mapped with sub-centimetre resolution. The direct surroundings of the river, including the riverbank itself, the vegetation, and the buildings are important factors for hydrodynamic modelling and for modelling the flooded area during a flood event.

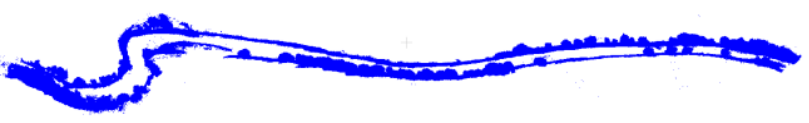

Figure 4. Lidar point cloud of a $1.5 \mathrm{~km}$ segment of the river Freiberger Mulde.

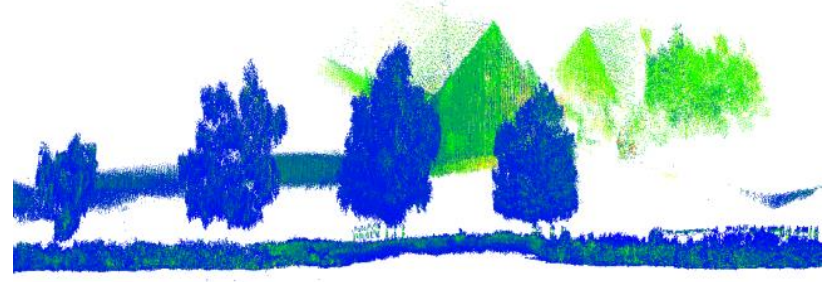

Figure 5. Details of the lidar point cloud. Colours show intensity. Only one bank of the river is shown, including trees and buildings in the background.

\subsection{Echo Soundings}

The best strategy for single beam echo sounder measurements with subsequent interpolation is to record cross sections with small gaps (Santillan et al., 2016). However, this was impractical, because the riverbanks would be out of the lidar's field of view. Therefore, a zigzag configuration was the best trade-off.

Interpolation of meandering rivers is influenced by an interaction of points, which are nearby in a world coordinate system, but not along the river. Echo sounder measurements need to be transformed into a flow-oriented coordinate system to minimize these influences (Merwade et al., 2016). Since there is no river centre line available, lidar points of the riverbanks have to be utilized for this transformation. The interpolation method plays a minor role compared to the data acquisition strategy, with Kriging performing best out of several interpolation methods (Krüger et al., 2018). Hence, Ordinary Kriging is applied.

Figure 6 includes the interpolation result as a depth coded point cloud. The river continues with a weir in the west, resulting in deep water in that area. As expected, there are also deeper parts where the river is narrower.

\subsection{Fusion with UAV data}

The point clouds of the river bathymetry and the geometry of the riverbanks are both geo-referenced. Figure 6 shows them both in UTM coordinates, together with a point cloud that was generated with SfM from UAV images. For further use in the EXTRUSO project, a DTM will be derived from that point cloud.

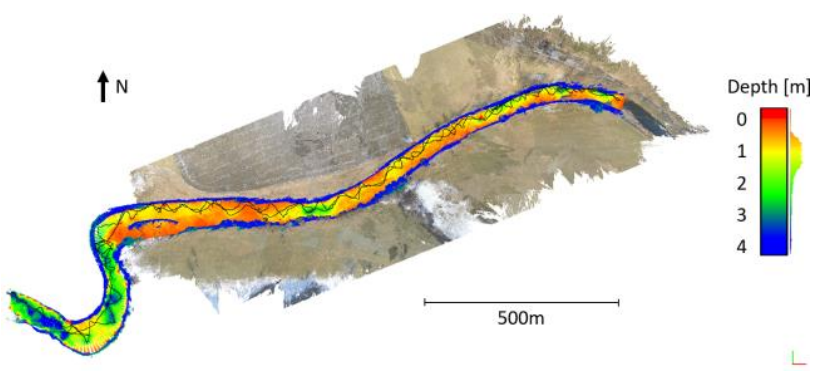

Figure 6. Results of a test surveys on the river Freiberger Mulde. Lidar points of the riverbanks are coloured in blue. The black line shows the UWV track in a zigzag configuration. The echo sounder data is depth coded. An UAV-based SfM point cloud maps the surroundings of the river.

\section{SUMMARY AND OUTLOOK}

This article shows the potential of boat-based river mapping for extreme event analysis and modelling. A design and calibration method for an unmanned water vehicle was presented. Point clouds of the river morphology recorded from an UWV can fill the gaps of UAV-based point cloud data. For further use for hydrodynamic modelling, DTMs have to be created. Hence, filtering the vegetation that covers the riverbanks has to be considered. The potential of using the multi-echo functionality of the mobile lidar for vegetation filtering should be evaluated in oncoming experiments. UWV based point clouds can also be used for post flood event analysis. Future work should include multi temporal acquisition and change detection of the river morphology before and after a flood event.

The accuracy of the acquired point clouds needs to be evaluated in more detail. Direct geo-referencing with IMU and GNSS leads to an error in the order of $50 \mathrm{~cm}$. RTK should be used to minimize that error. Additionally, control points can be added. Adapting the calibration method with a boresight alignment and lever arm calibration and by designing a calibration method that includes the echo sounder will also improve the accuracy, especially when larger distances will be surveyed. The interpolation results for bathymetry highly depends on point density. In order to improve the interpolation accuracy, an acquisition strategy with higher zigzag frequency should be applied. Using a depth sensor with a larger field of view could also densify the point cloud. A triangulation sensor applying a laser line and an underwater camera will be tested in coming experiments.

\section{ACKNOWLEDGEMENTS}

These investigations are part of the research project "Extreme events in small and medium catchments (EXTRUSO)" funded by the EU ESF 'Junior Research Groups' program under grant no. 100270097. The UAV point cloud was provided by the Chair of Geoinformatics at the TU Dresden and was developed in the research project "BOOT-Monitoring", which is part of the funding program 'ReWaM' by the German Federal Ministry of Education and Research.

\section{REFERENCES}

Borga, M., Gaume, E., Creutin, J. D., and Marchi, L., 2008. Surveying flash floods: gauging the ungauged extremes. $\begin{array}{lll}\text { Hydrological } & \text { processes, } & 22(18),\end{array}$ https://doi.org/10.1002/hyp.7111 
Krüger, R., Karrasch, P., and Bernard, L., 2018. Evaluating Spatial Data Acquisition and Interpolation Strategies for River Bathymetries. A. Mansourian et al. (eds.), Geospatial Technologies for All, Springer International Publishing, 3-24, https://doi.org/10.1007/978-3-319-78208-9_1

Mader, D., Westfeld, P., and Maas, H.-G., 2014. AN INTEGRATED FLEXIBLE SELF-CALIBRATION APPROACH FOR 2D LASER SCANNING RANGE FINDERS APPLIED TO THE HOKUYO UTM-30LX-EW. The International Archives of the Photogrammetry, Remote Sensing and Spatial Information Sciences XL-5, 385-393. https://doi.org/10.5194/isprsarchives-XL-5-385-2014

Merwade, V. M., Maidment, D., R., and Goff, J. A., 2006. Anisotropic considerations while interpolating river channel bathymetry. Journal of Hydrology 332, 732-741. https://doi.org/10.1016/j.jhydrol.2006.06.018

Mueller, E. N., and Pfister, A., 2011. Increasing occurrence of high-intensity rainstorm events relevant for the generation of soil erosion in a temperate lowland region in Central Europe. Journal of Hydrology, 411(3), 266-278, https://doi.org/10.1016/j.jhydrol.2011.10.005

Santillan, J. R., Serviano, J. L., Makinano-Santillan, M., and Marqueso, J. T., 2016. INFLUENCE OF RIVER BED ELEVATION SURVEY CONFIGURATIONS AND INTERPOLATION METHODS ON THE ACCURACY OF LIDAR DTM-BASED RIVER FLOW SIMULATIONS. The International Archives of the Photogrammetry, Remote Sensing and Spatial Information Sciences XLII-4/W1, 225-235. https://doi.org/10.5194/isprs-archives-XLII-4-W1-225-2016 